\title{
The history and future of the Local and Loop I bubbles
}

\author{
D. Breitschwerdt ${ }^{1}$ and M. A. de Avillez ${ }^{1,2}$ \\ 1 Institut für Astronomie, Universität Wien, Türkenschanzstraße 17, 1180 Wien, Austria \\ e-mail: breitschwerdt@astro.univie.ac.at \\ 2 Department of Mathematics, University of Évora, R. Romão Ramalho 59, 7000 Évora, Portugal \\ e-mail: mavillez@galaxy.lca.uevora.pt
}

Received 9 February 2006 / Accepted 30 March 2006

ABSTRACT

\begin{abstract}
Context. The Local and Loop I superbubbles are the closest and best investigated supernova (SN) generated bubbles and serve as test laboratories for observations and theories of the interstellar medium.

Aims. Since the morphology and dynamical evolution of bubbles depend on the ambient density and pressure distributions, a realistic modelling of the galactic environment is crucial for a detailed comparison with observations.

Methods. We have performed 3D high resolution (down to $1.25 \mathrm{pc}$ on a kpc-scale grid) hydrodynamic simulations of the Local Bubble (LB) and the neighbouring Loop I (L1) superbubble in a realistically evolving inhomogeneous background ISM, disturbed already by SN explosions at the Galactic rate for $200 \mathrm{Myr}$ before the LB and L1 are generated. The LB is the result of 19 SNe occurring in a moving group, which passed through the present day local HI cavity.

Results. We can reproduce (i) the OVI column density in absorption within the LB in agreement with CoPERNICUS and recent FUSE observations, giving $N_{\text {OVI }}<2 \times 10^{13} \mathrm{~cm}^{-2}$ and $N_{\text {OVI }}<7 \times 10^{12} \mathrm{~cm}^{-2}$, respectively; (ii) the observed sizes of the Local and Loop I superbubbles; (iii) the interaction shell between LB and L1, discovered with ROSAT; (iv) constrain the age of the LB to be $14.5 \pm_{0.4}^{0.7} \mathrm{Myr}$; (v) predict the merging of the two bubbles in about $3 \mathrm{Myr}$, when the interaction shell starts to fragment; (vi) the generation of blobs like the Local Cloud as a consequence of a dynamical instability.

Conclusions. We find that evolving superbubbles strongly deviate from idealised self-similar solutions due to ambient pressure and density gradients, as well as due to turbulent mixing and mass loading. Hence, at later times the hot interior can break through the surrounding shell, which may also help to explain the puzzling energy "deficit" observed in LMC bubbles.
\end{abstract}

Key words. hydrodynamics - shock waves - ISM: general - ISM: bubbles - ISM: structure - ISM: kinematics and dynamics

\section{Introduction}

Our solar system is embedded in an HI cavity extending about $200 \mathrm{pc}$ in the Galactic plane and roughly $600 \mathrm{pc}$ perpendicular to it, according to NaI absorption line studies towards background stars (Lallement et al. 2003). For a long time it has been known that at least part of this so-called Local Bubble (LB) shows diffuse emission in soft X-rays (cf. McCammon \& Sanders 1990). Ever since the LB has been the subject of intensive studies in radio, UV, EUV and soft X-rays, as well as of analytical and numerical modelling (e.g. Cox \& Anderson 1982; Breitschwerdt \& Schmutzler 1994; Smith \& Cox 2001). However, due to the wealth of data and the inherent complex structure of the LB, all models have severe shortcomings, concerning either the dynamical evolution or the model spectra, in some cases both. In particular models based on thermal conduction usually fail to reproduce the observed low OVI absorption column density of $\sim 1.6 \times 10^{13} \mathrm{~cm}^{-2}$ in the Local Bubble as inferred from a reanalysis of COPERNICUS data (Shelton \& Cox 1994). One severe restriction in all the models, which consider the LB to be the result of SN explosions, viz. the expansion into a homogeneous ambient medium, has been removed in the simulations presented here. As a corollary, the LB evolution has to be performed jointly with that of the Loop I (L1) superbubble. ROSAT PSPC observations, revealing a coherent X-ray shadow towards L1, led to the suggestion (Egger \& Aschenbach 1995) that the LB is in close contact with the expanding neighbouring L1 superbubble. L1 thus originated from multi-supernova explosions in the Sco Cen cluster, with the North Polar Spur being part of its shock illuminated outer shell.

It has also been suggested that part of the soft X-ray emission could be of very local origin, generated by charge exchange reactions between solar wind ions and heliospheric (e.g. Cravens 2000) or even atmospheric plasma. However, even in the most favourable case of all emission being very local in a certain direction, there is always missing flux along other lines of sight, in particular perpendicular to the Galactic plane.

In this letter we describe for the first time a realistic evolutionary scenario for the origin and evolution of the Local Bubble jointly with the L1 superbubble. The crucial differences as compared to previous models are: (i) a realistic background medium, that has been pre-structured by previous generations of SN explosions, which is therefore very inhomogeneous, both in density and pressure distribution; (ii) taking into account the time sequence and locations of SN explosions, which generate the two bubbles, calculated according to the mass distribution of extinct members of a moving group and the Sco Cen cluster, respectively, both derived from a Galactic initial mass function (IMF) normalised to still existing low mass stars; and (iii) to calculate the density and temperature structure of the LB and L1 
in 3D with high resolution in order to sample OVI along many different lines of sight and compare them to observations.

This letter is organised as follows: Sect. 2 describes details of the model setup, in Sect. 3 our results with respect to bubble evolution and comparison to observations are discussed, and we close with a general discussion and conclusions in Sect. 4.

\section{Model and simulations}

We use a 3D parallelised adaptive mesh refinement (AMR) hydrocode to track small scale structures down to $1.25 \mathrm{pc}$, where necessary, and we follow the LB and L1 evolution in an ISM, driven by $\mathrm{SNe}$ types $\mathrm{Ia}, \mathrm{Ib}+\mathrm{c}$ and II occurring at the Galactic rate (taken from Cappellaro et al. 1999), on a Cartesian grid of $0 \leq(x, y) \leq 1 \mathrm{kpc}$ size in the Galactic plane, and $-10 \leq z \leq$ $10 \mathrm{kpc}$ perpendicular to it. This is an extension of the SN-driven ISM model of Avillez (2000), fully tracking the time-dependent evolution of the large scale disk and the Galactic fountain. The gravitational field of the stellar disk is adopted from Kuijken \& Gilmore (1989). Further processes include radiative cooling, assuming an optically thin gas in collisional ionisation equilibrium (CIE, i.e. ionisation rate by collisions between electrons and ions is balanced by radiative recombination rate) using the cooling functions of Sutherland \& Dopita (1993) for $T \geq 10^{4} \mathrm{~K}$ and Dalgarno \& McCray (1972) for $T<10^{4} \mathrm{~K}$, with a temperature cut-off at $10 \mathrm{~K}$, and uniform heating due to starlight varying with height $z$ (Wolfire et al. 1995). Galactic OB associations form in regions with density $n \geq 10 \mathrm{~cm}^{-3}$ and temperature $T \leq 100 \mathrm{~K}$, with the number, masses and main sequence life times of the stars in the association being determined from the IMF. All OB stars are allowed to drift away from their parent associations with random velocities assigned according to observations, before exploding either in clusters or in the field with a canonical energy of $10^{51} \mathrm{erg}$.

The LB itself is carved by 19 successive explosions of the subgroup B1 of Pleiades, of which some low mass stars are now Sco Cen cluster members according to the model of Berghöfer \& Breitschwerdt (2002, henceforth BB02). The adjacent L1 bubble is the result of explosions of Sco Cen cluster members (see Egger 1998). Applying an IMF for OB associations (Massey et al. 1995) and following BB02, we find that about $39 \mathrm{SNe}$ have occurred in L1 until now, and $38 \mathrm{SN}$ candidates are expected to explode within the next $13 \mathrm{Myr}$. In contrast, the LB is extinct now, the last explosion having occurred about 0.5 Myr ago. Main sequence life times are calculated from Stothers (1972). As initial conditions for the present run we used data cubes of an evolved SN driven ISM, in which global dynamical equilibrium has been established (cf. Avillez \& Breitschwerdt 2004 (henceforth AB04), 2005a). As the ISM structure reveals a typical pattern size of a few hundred pc (Avillez \& Breitschwerdt 2005b, henceforth $\mathrm{AB} 05$ ), we chose arbitrarily a site with a sufficient amount of cold HI mass to form the Sco Cen cluster, here located at $(375,400)$ pc (cf. Fig. 1). Then we followed the trajectory of the Pleiades moving group B1, whose SNe in the LB went off along a path crossing the location at $(175,400) \mathrm{pc}$, chosen to match the present observed distances from the Sun and the Sco Cen. Due to small peculiar motions of the local gas, the LB will be tied to the local standard of rest (LSR; for details of the moving group kinematics see BB02). Periodic boundary conditions are applied along the four vertical boundary faces, while outflow boundary conditions are imposed at the top $(z=10 \mathrm{kpc})$ and bottom $(z=-10 \mathrm{kpc})$ boundaries. The simulation time of the LB and L1 evolution run was an additional $30 \mathrm{Myr}$.
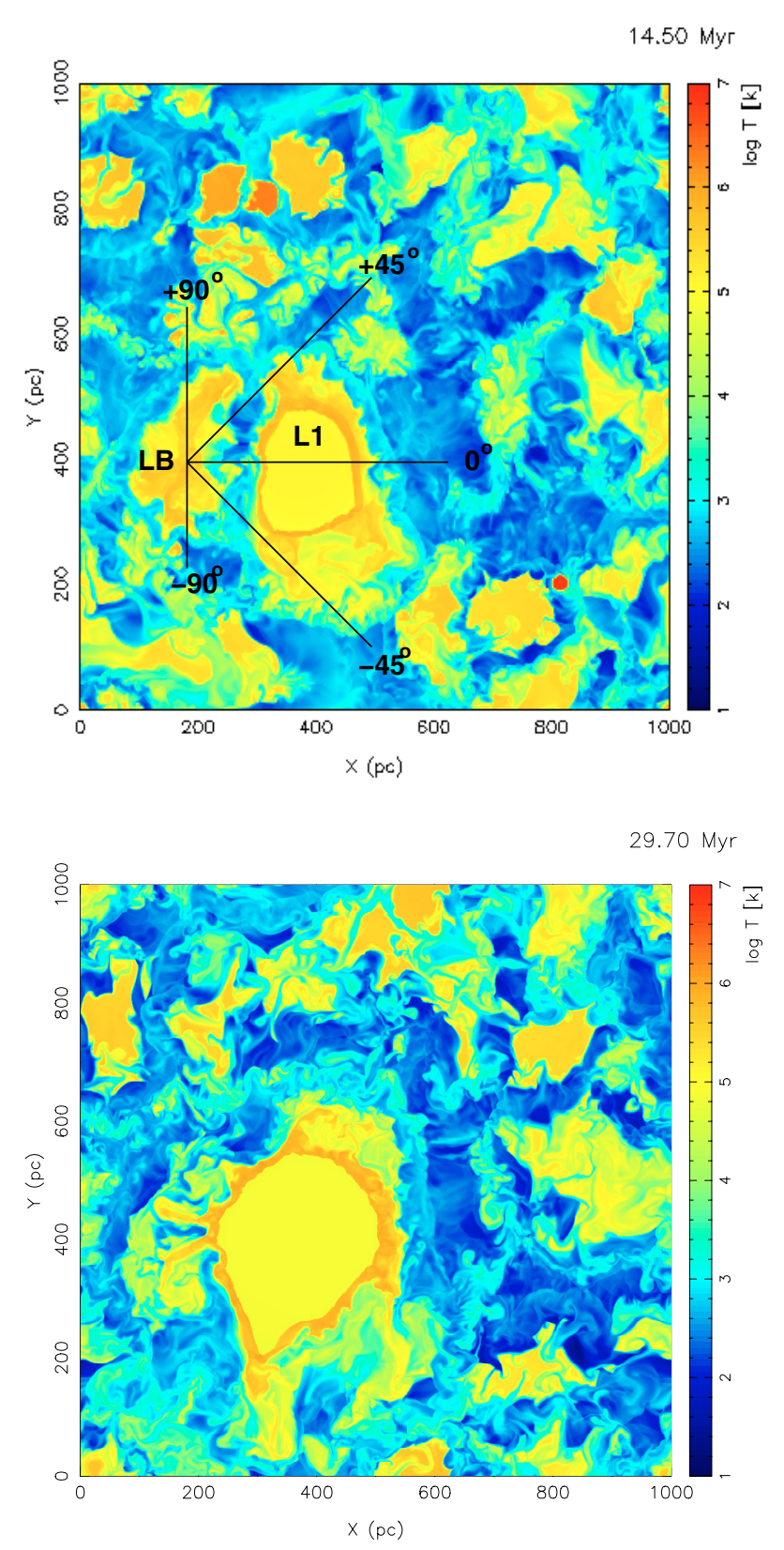

Fig. 1. Top: colour coded temperature map in the range $10 \leq T \leq 10^{7} \mathrm{~K}$ for a slice through the data cube (representing the Galactic midplane) of a 3D LB high resolution simulation representing the present time (i.e. 14.5 Myr after the first explosion) with the LB centred at $(175,400) \mathrm{pc}$ and L1 shifted $200 \mathrm{pc}$ to the right. Bottom: same, showing the "future" of the LB and L1 at $t=29.7$ Myr. Note that part of the LB has merged with the ISM, part has been engulfed by L1.

\section{Results}

\subsection{Evolution of the Local and Loop I superbubbles}

The present runs were started after global dynamical equilibrium, specifically the Galactic Fountain, had been established (i.e. after $200 \mathrm{Myr}$ of evolution for a Galactic SN rate; for details, see AB04). The locally enhanced SN rates produce coherent LB and L1 structures within a highly disturbed and still evolving background medium (due to ongoing star formation). Successive explosions heat and pressurise the cavities, which at first look smooth, but develop internal structure after some time; for the LB this occurs at $t>8 \mathrm{Myr}$ after the first explosion. 
$14.50 \mathrm{Myr}$

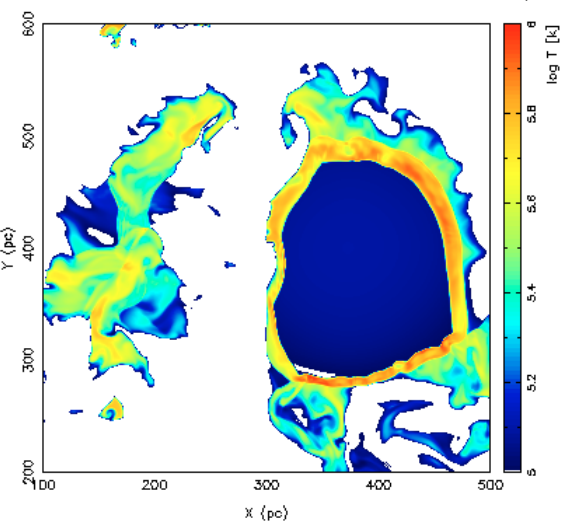

$14.80 \mathrm{Myr}$

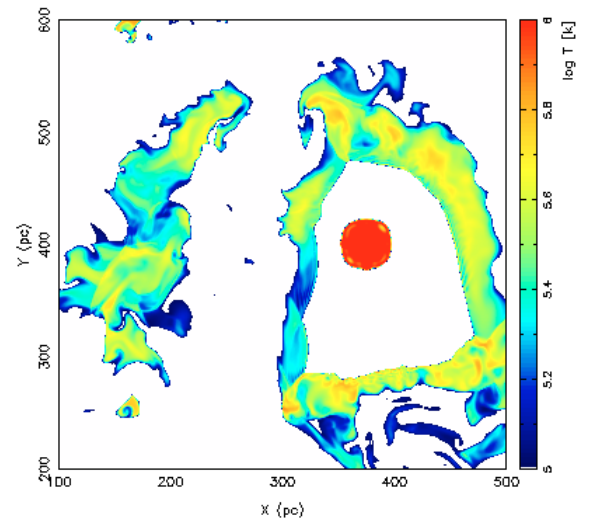

$14.60 \mathrm{Myr}$

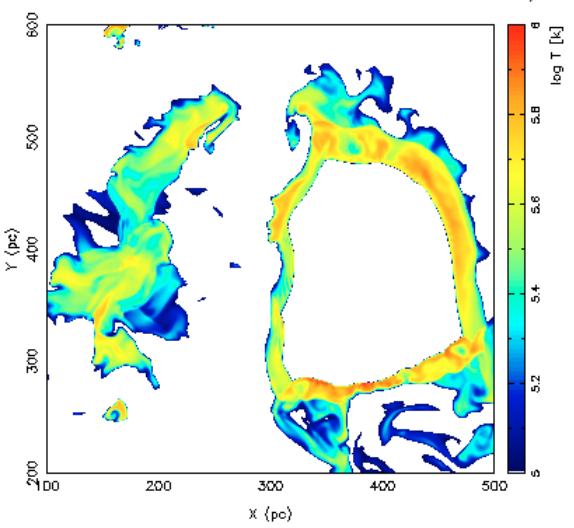

$14.90 \mathrm{Myr}$

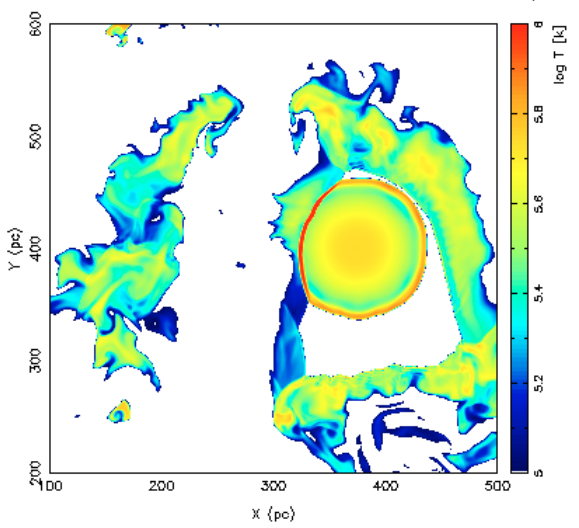

$14.70 \mathrm{Myr}$

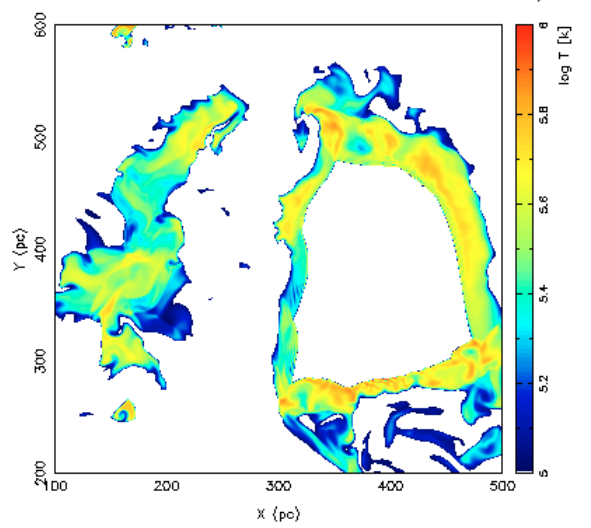

$15.00 \mathrm{Myr}$

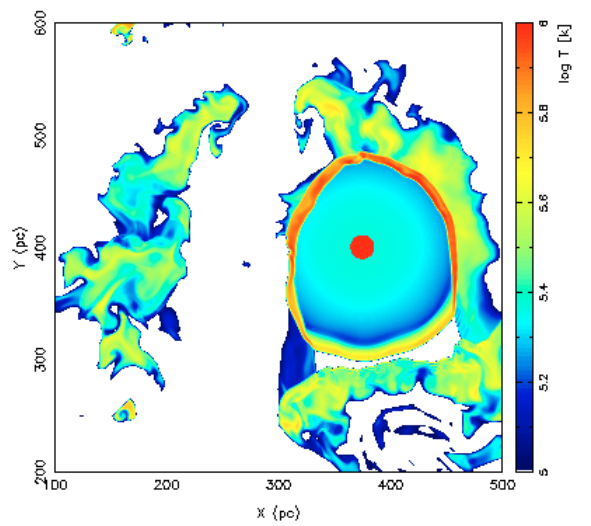

Fig. 2. Temperature distribution in the Galactic plane in the range $10^{5} \leq T \leq 10^{6} \mathrm{~K}$ of the LB and L1 region, top: at $t=14.5$ (left), $t=14.6$ (middle) and $t=14.7 \mathrm{Myr}$ (right), and bottom: at $t=14.8$ (left), $t=14.9$ (middle) and $t=15.0 \mathrm{Myr}$ (right), after the first LB explosion. Note that in L1, SN explosions are still occurring (see bottom panel), while the LB is extinct.

After $\sim 14.5 \mathrm{Myr}$ (present time) $19 \mathrm{SNe}$ have exploded inside the LB cavity, which becomes progressively elongated and reaches a size of 180 by $220 \mathrm{pc}$ in the Galactic midplane (Fig. 1 top), due to the increasing influence of ambient density and pressure gradients. Although magnetic fields and cosmic rays have an influence on bubble evolution by tension and pressure forces, it seems unlikely that our results will change significantly, because in the LB the last SN went off only $0.5 \mathrm{Myr}$ ago, and Loop I is still active. If the bubble pressure decreases markedly there may however be some deviations. Both bubbles are bounded by shells whose interaction already generates Rayleigh-Taylor instabilities due to a larger pressure in L1 with respect to the LB, in agreement with a linear stability analysis of Breitschwerdt et al. (2000). After becoming nonlinear this will lead to the formation of cloudlets, which will travel towards the solar system from the Sco Cen region, in agreement with observations of local clouds with measured velocities of about $26 \mathrm{~km} \mathrm{~s}^{-1}$. On the basis of our simulations we further predict that the interaction shell will break up in 3 Myr from now, allowing mass transfer of hot gas from $\mathrm{L} 1$ to the $\mathrm{LB}$, and in $\sim 15 \mathrm{Myr}$ from now the bubbles will have merged (see Fig. 1 bottom).

In CIE, OvI is most abundant at $T \sim 3 \times 10^{5} \mathrm{~K}$, whereas soft X-Ray emission of a thermal plasma requires a temperature of $\sim 10^{6} \mathrm{~K}$. Figure 2 shows the temperature distribution in the critical range, $10^{5} \leq T \leq 10^{6} \mathrm{~K}$, during the critical time, $14.5 \leq t \leq 15.0 \mathrm{Myr}$, when the LB plasma starts cooling down. It is obvious that between 14.5 and $14.7 \mathrm{Myr}$, cooler (OVI absorbing) and hotter (X-ray emitting) gas co-exist. This is a direct result of the turbulent and inhomogeneous density and temperature structure in realistically evolved superbubbles. Turbulent mixing generates gas in the whole thermally unstable regime between $10^{5}-10^{6} \mathrm{~K}$. While the amount of OVI in the LB has been measured fairly accurately (see Sect. 3.2), the source of the soft X-ray emission is more elusive. First of all, it is more difficult to localise, as there are most likely no dense clouds inside the LB, which can be used for shadowing experiments, thus allowing us to separate back- and foreground emission. Secondly, a yet uncertain amount of soft X-rays will be produced locally by solar wind charge exchange reactions with heliospheric plasma (Cravens 2000). And thirdly, the LB plasma need not be in CIE. It has been shown that if a plasma undergoes fast adiabatic expansion its kinetic temperature will drop, but recombination can not follow fast enough, thus mimicking an "overionised" plasma (similar to the solar wind), where recombination is delayed. In essence, the gas could be at a few times $10^{5} \mathrm{~K}$, but still emit copiously in soft X-rays (Breitschwerdt \& Schmutzler 1994). We are currently working on these non-equilibrium ionisation (NEI) models, and will present results in a forthcoming paper.

\subsection{O VI distribution in the solar neighbourhood}

We have determined the amount of OVI along the lines of sight (LOS) for an ISM plasma in CIE with solar abundances (Anders $\&$ Grevesse 1989; for details see AB05). To measure the OVI column density distribution inside LB and L1, we took 91 LOS extending from the Sun and crossing L1 (the hot pressured region $200 \mathrm{pc}$ to the right of the LB) from an angle of $-45^{\circ}$ to $+45^{\circ}$ as marked in the top panel of Fig. 1. Figure 3 (top) shows the 

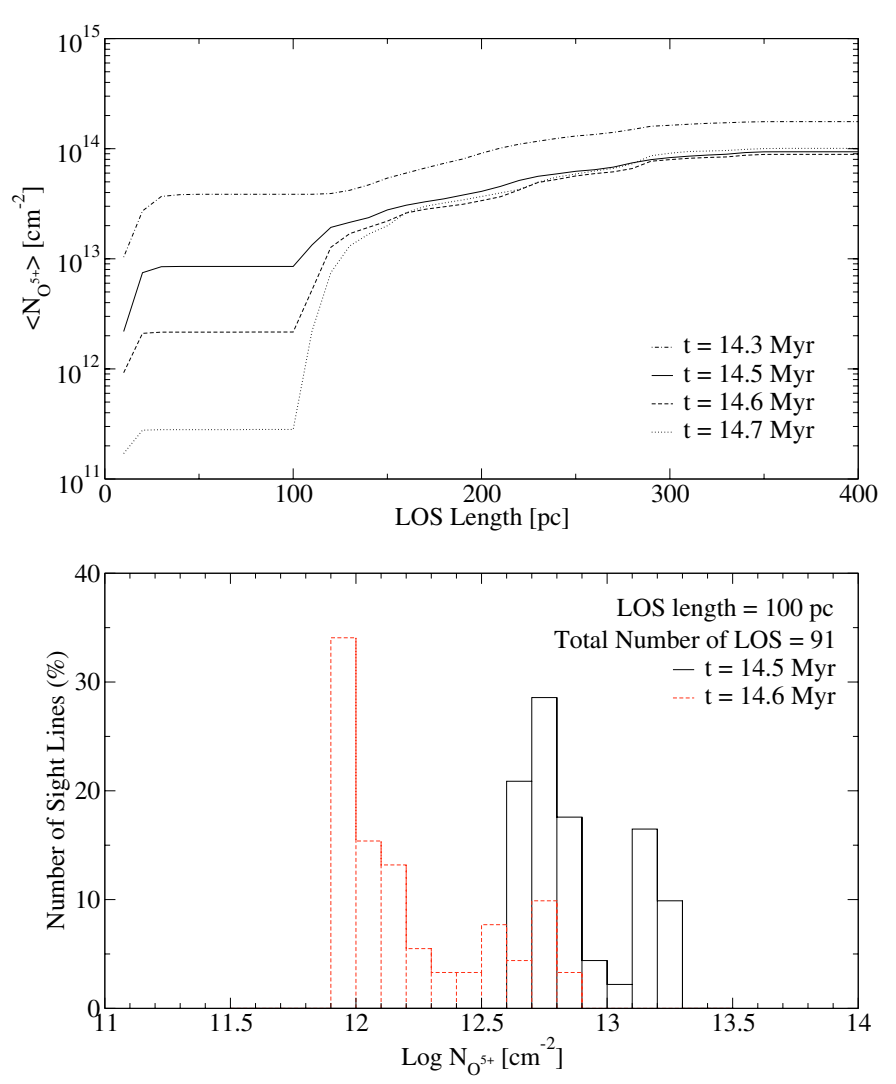

Fig. 3. Top: averaged OvI column densities (over 91 LOS between $\pm 45^{\circ}$ as marked in Fig. 1 top ) as a function of LOS path length at $14.3 \leq t \leq$ 14.7 Myr of Local and L1 bubbles evolution. Bottom: histogram of the percentage of LOS as a function of $N(\mathrm{OVI})$ within the LB at $t=14.5$ and 14.6 Myr.

history (for $14.3 \leq t \leq 14.7 \mathrm{Myr}$ ) of the variation of the LOS averaged OVI column densities, $\langle N(\mathrm{OVI})\rangle$, with distance along these LOS. They decrease steeply with time after the last $\mathrm{SN}$ in the LB has exploded, from $4 \times 10^{13}$ to $3 \times 10^{11} \mathrm{~cm}^{-2}$, because no further heating is taking place. For LOS sampling gas from outside the LB (i.e., $l_{\mathrm{LOS}}>100 \mathrm{pc}$ ) we have $\langle N(\mathrm{OVI})\rangle>$ $2 \times 10^{13} \mathrm{~cm}^{-2}$. The histograms of column densities obtained in the $91 \mathrm{LOS}$ for $t=14.5$ and 14.6 Myr (Fig. 3, bottom) show that for $t=14.6 \mathrm{Myr}$ all the LOS have column densities smaller than $10^{12.9} \mathrm{~cm}^{-2}$, while for $t=14.5 \mathrm{Myr} 67 \%$ of the lines have column densities smaller than $10^{13} \mathrm{~cm}^{-2}$ and about $50 \%$ of the lines have $N(\mathrm{OVI}) \leq 7.9 \times 10^{12} \mathrm{~cm}^{-2}$. The maximum column density is $1.78 \times 10^{13} \mathrm{~cm}^{-2}$ in excellent agreement with the observations discussed in Savage \& Lehner (2006), which is 1.1 times the average value of $1.6 \times 10^{13} \mathrm{~cm}^{-2}$ inferred by Shelton \& Cox (1994) from a reanalysis of CoPERNICUS absorption line data (cf. Jenkins 1978).

In Fig. 4 we compare FUSE data by Oegerle et al. (2005; triangles) and Savage \& Lehner (2006; circles) with simulated minimum (red squares), maximum (green squares) and averaged (blue squares) column density of OVI measurements along the 91 LOS outlined in Fig. 1 (top). It can be clearly seen in Fig. 4 that for $t=14.5 \mathrm{Myr}$ the calculated N(OVI) distribution in the LB is similar to that observed with FUSE. These results allow us to constrain the age of the LB to be $14.5 \pm_{0.4}^{0.7} \mathrm{Myr}$, and capture at the same time all the relevant LB properties, such as size, age, dynamical instabilities in the interaction shell, amongst others.

Successive blast waves advance very fast through the lowdensity cavity, but slow down considerably later, as they run into

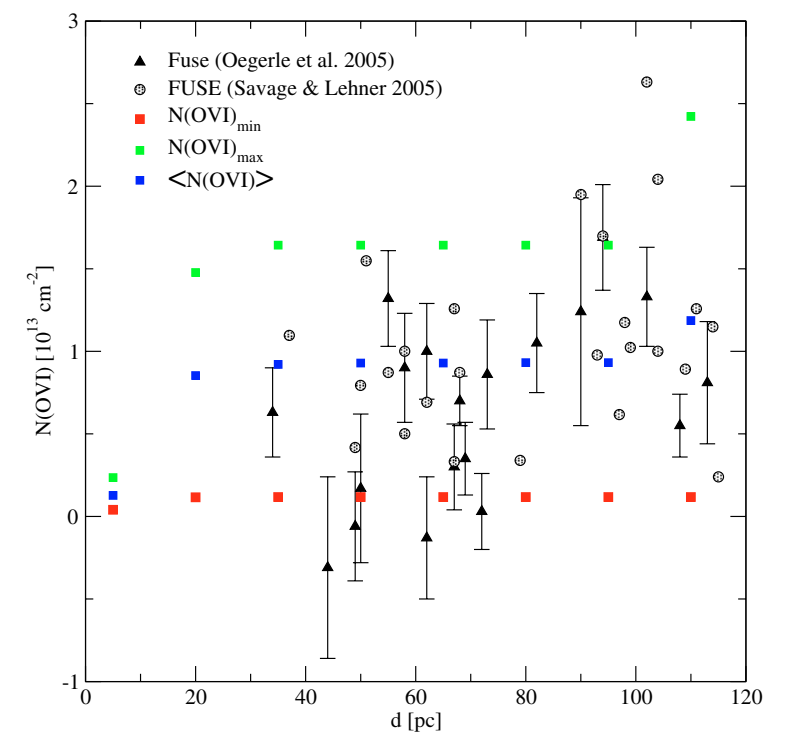

Fig. 4. Comparison between FUSE (triangles: Oegerle et al. 2005; circles: Savage \& Lehner 2006) OVI column densities with the calculated minimum, maximum and average column densities along the same 91 lines of sight (at $t=14.5 \mathrm{Myr}$ ) pointing towards L1 as shown in Fig. 1 (top). Negative values on the ordinate derive from essentially zero equivalent absorption line widths, i.e. non-detections, due to the noise in the spectrum (cf. Oegerle et al. 2005).

the dense shell. As a consequence an asymmetric reverse shock leads to shear flow and turbulence inside the cavity, with a largest eddy size of a fraction of the bubble diameter, typically $l \leq 75 \mathrm{pc}$ (Avillez \& Breitschwerdt 2006). Thus the turnover time scale is about $\tau_{\mathrm{m}} \sim l / c_{\mathrm{s}} \leq 3.7 \times 10^{5} \mathrm{yr}$, for an averaged sound speed of $c_{\mathrm{S}} \approx 200 \mathrm{~km} \mathrm{~s}^{-1}$ after a SN explosion. As the last SN occurred about $0.5 \mathrm{Myr}$ ago, we expect that $\mathrm{SN}$ ejected oxygen inside the $\mathrm{LB}$ at present time should have a rather uniform $\mathrm{O} / \mathrm{H}$ distribution, a result that is also confirmed by FUSE observations of the $\mathrm{O} / \mathrm{H}$ ratio (Moos et al. 2002).

\section{Discussion and conclusions}

According to Cox (2004) a crucial test for any viable LB model is the reproduction of the measured amount of OVI. By embedding the LB into a typical inhomogeneous ISM, we obtain bubbles, which have more internal structure, exhibit on average lower pressure, consistent with that observed in the local clouds, show substantial mass loading (cf. Dyson \& Hartquist 1987), and are more susceptible to break-up of the surrounding shell (both in the disk and perpendicular to it), than was reported by previous authors. In fact there is some evidence from stellar absorption line studies towards high latitude stars, that the LB might be a Local Chimney (Welsh et al. 1999). Break-out of gas flows from superbubbles might also be a clue to the solution of the "energy problem" observed in LMC bubbles (Oey \& García-Segura 2004), which seem to be systematically too small compared to similarity solutions.

Finally, we would like to stress that our LB evolution model has the highest resolution so far obtained, showing structures down to $1.25 \mathrm{pc}$. We have repeated our runs with $0.625 \mathrm{pc}$ finest resolution to confirm that our results are not resolution dependent. This will be a prerequisite to model in a next step also the EUV and soft X-ray emission, including the full non-equilibrium ionisation structure. These calculations are underway. 
Acknowledgements. This work has been partially funded by FCT under PESO/P/PRO/40149/2000 to MAdeA and DB. We thank the two referees, Don Cox and an anonymous one, for their constructive criticism and helpful comments.

\section{References}

Anders, E., \& Grevesse, N. 1989, Geochim. Cosmochim. Acta, 53, 197 Avillez, M. A. 2000, MNRAS, 315, 479

Avillez, M. A., \& Breitschwerdt, D. 2004, A\&A, 425, 899 (AB04)

Avillez, M. A., \& Breitschwerdt, D. 2005a, A\&A, 436, 585

Avillez, M. A., \& Breitschwerdt, D. 2005b, ApJ, 634, L65 (AB05)

Avillez, M. A., \& Breitschwerdt, D. 2006, ApJL, submitted

Berghöfer, T., \& Breitschwerdt, D. 2002, A\&A, 390, 299 (BB02)

Breitschwerdt, D., \& Schmutzler, T. 1994, Nature, 371, 774

Breitschwerdt, D., Egger, R., \& Freyberg, M. J. 2000, A\&A, 361, 303

Cappellaro, E., Evans, R., \& Turatto, M. 1999, A\&A, 351, 459

Cox, D. P. 2004, Ap\&SS, 289, 469

Cox, D. P., \& Anderson, P. R. 1982, ApJ, 253, 268

Cravens, T. E. 2000, ApJ, 532, L153

Dalgarno, A., \& McCray, R. A. 1972, ARA\&A, 10, 375
Dyson, J. E., \& Hartquist, T. W. 1987, MNRAS, 228, 453

Egger, R. 1998, in The Local Bubble and Beyond, ed. D. Breitschwerdt, M. J. Freyberg, \& J. Trümper (Berlin: Springer-Verlag), Lecture Notes in Physics, 506, 287

Egger, R., \& Aschenbach, B. 1995, A\&A, 294, L25

Jenkins, E. B. 1978, ApJ, 219, 845

Kuijken, K., \& Gilmore, G. 1989, MNRAS, 239, 651

Lallement, R., Welsh, B. Y., Vergely, J. L., Crifo, F., \& Sfeir, D. 2003, A\&A, 411, 447

Massey, P., Johnson, K. E., \& Degioia-Eastwood, K. 1995, ApJ, 454, 151

McCammon, D., \& Sanders, W. T. 1990, ARA\&A, 28, 657

Moos, H. W., Sembach, K. R., Vidal-Madjar, A., et al. 2002, ApJS, 140, 3

Oegerle, W. R., Jenkins, E. B., Shelton, R. L., Bowen, D. V., \& Chayer, P. 2005, ApJ, 622, 377

Oey, M. S., \& García-Segura, G. 2004, ApJ, 613, 302

Savage, B. D., \& Lehner, N. 2006, ApJS, 162, 134

Shelton, R., \& Cox, D. P. 1994, ApJ, 434, 599

Smith, R. K., \& Cox, D. P. 2001, ApJS, 134, 283

Stothers, R. 1972, ApJ, 175, 431

Welsh, B. Y., Sfeir, D. M., Sirk, M. M., \& Lallement, R. 1999, A\&A, 352, 308

Sutherland, R. S., \& Dopita, M. A. 1993, ApJS, 88, 253

Wolfire, M. G., McKee, C. F., Hollenbach, D., Tielens, A. G. G. M., \& Bakes, E. L. O. 1995 , ApJ, 443, 152 